\title{
Humidity and Pressure Regulation in a PEM Fuel Cell Using a Gain-Scheduled Static Feedback Controller
}

\author{
Amey Y. Karnik, Jing Sun, Fellow, IEEE, Anna G. Stefanopoulou, and Julia H. Buckland
}

\begin{abstract}
In this paper, the pressure difference between the anode and cathode compartments of a polymer electrolyte membrane (PEM) fuel cell stack is regulated along with the anode and cathode humidities using an anode recirculation system. The pressure regulation requirement stems from membrane safety considerations. The regulation of average humidities in the two compartments is a necessary (although not a sufficient) requirement for stack water management. Two actuators in the anode recirculation system are considered, namely the dry hydrogen flow and the anode back pressure valve. These actuators are adjusted using a static output feedback controller that relies on pressure and humidity measurements on the anode side of the fuel cell stack. As the water mass dynamics and the characteristics of the water transport through the PEM are significantly different between subsaturated conditions (water is present only in vapor phase) and saturated conditions (liquid water along with water vapor), we show that the performance of the static output feedback controller with a fixed set of gains for subsaturated condition deteriorates significantly under a saturated condition. A gain-scheduled controller is therefore developed to compensate for a water-vapor saturated cathode condition. Analysis and simulation provide insights on some of the design and implementation issues for the gain-scheduled output feedback system.
\end{abstract}

Index Terms-Fuel cells, humidity control, output feedback, pressure control.

\section{INTRODUCTION}

$\mathbf{F}$ UEL CELL-BASED powertrain systems promise to deliver cleaner and more efficient vehicles [1]. In order to provide a performance comparable to conventional powertrain systems, the polymer electrolyte membrane (PEM) fuel cell powertrain system has to meet stringent power and durability requirements under demanding operating conditions encountered in automotive applications. Meeting these challenges requires substantial improvement in the materials and construction of the fuel cells. Equally important is the synergetic integration of the fuel cell "system," which consists of the fuel cell stack and the auxiliary components such as the reactant

Manuscript received August 03, 2007; revised November 20, 2007. Manuscript received in final form April 03, 2008. First published July 25, 2008; current version published February 25, 2009. This work was supported by Ford Motor Company through the University Research Program. Recommended by Associate Editor K. Hunt.

A. Y. Karnik and J. H. Buckland are with the Powertrain Controls R\&A Department, Ford Motor Company, Dearborn, MI 48124 USA (e-mail: akarnik@ford.com; jbucklan@ford.com).

J. Sun is with the Naval Architecture and Marine Engineering Department, 211 Naval Architecture and Marine Engineering, Ann Arbor, MI 48109 USA (e-mail: jingsun@umich.edu).

A. G. Stefanopoulou is with the Mechanical Engineering Department, University of Michigan, Ann Arbor, MI 48109-2121 USA (e-mail: annastef@ umich.edu)

Digital Object Identifier 10.1109/TCST.2008.924562 supply systems, the temperature control unit and the water management systems [2].

The performance and longevity of a PEM fuel cell (PEMFC) system depend upon a water management system that balances the water generated and the water introduced into the system by the humidified inlet flows, with the water removed from the system [3], [4]. In this paper, an important task of the overall water management goal is addressed. Namely, the humidity regulation in the anode and cathode compartments of the PEM fuel cell is accomplished by adjusting the anode water removal and the cathode inlet humidity according to the load, that is, the stack current drawn. Although we limit the scope of this paper to the control of the anode and cathode humidities in the average sense, regulating the average humidities can be viewed as controlling the boundary conditions to the spatial distribution of water within the stack.

The average humidities in both the anode and the cathode compartments of the fuel cell stack should be high enough to allow sufficient humidification of the membrane separating the two compartments. In addition, the regulation of the anode and cathode humidities during transients in load is necessary to avoid excessive liquid water accumulation in the stack which can impede the reactant flows. The lack of sensors for measuring the amount of liquid water in a compartment in addition to a change in the system dynamics between the conditions with and without liquid water in the compartments make the water management an interesting control problem.

In this paper, we explore the use of feedback control to address the transient water management problem of a PEMFC system. For the system under investigation, the required cathode inlet humidity is provided by flowing the air supplied to the cathode of the fuel cell stack through a humidifier. Meanwhile, the other mechanism used in water management, that is, the anode water removal is rendered by the unconsumed fuel (hydrogen) leaving the anode. The unconsumed fuel is recirculated to the anode inlet using a device such as a blower or an ejector [5] after the liquid water is extracted from the anode exit flow using a condenser with a knock-out drum.

A PEMFC system with an ejector-based anode recirculation loop is shown in Fig. 1. The ejector acts as a pumping device to overcome the adverse pressure difference between the anode exit and the anode inlet. In addition, the ejector mixes the recirculated flow with dry hydrogen supplied from the fuel source. While the ejector is a key element for anode recirculation, it is the condenser in the recirculation path that provides water removal. The anode water removal rate is controlled by regulating the recirculation flow using a back pressure valve placed between the condenser and the anode exit.

The two water-management mechanisms described above are used to regulate the desired humidity conditions in the anode 


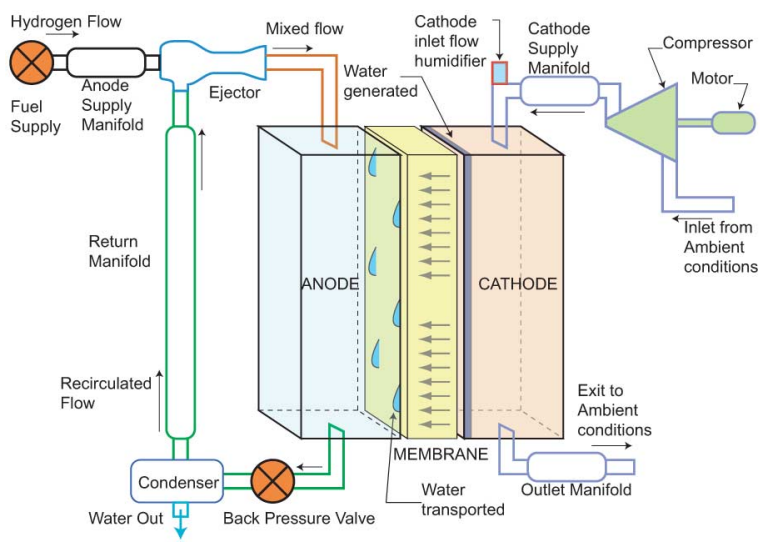

Fig. 1. Schematic of the fuel cell system.

and cathode. Apart from humidity regulation, it is important to avoid a large pressure difference across the membrane by regulating the anode pressure to follow the cathode pressure. We present a design methodology to develop feedback controllers that meet both the water management requirements and the pressure regulation requirements in the presence of load transients. To allow modularity in the design of the stack auxiliary system, we consider the scenario where the transient requirements are addressed using only the actuators in the anode recirculation system, namely the fuel supply and the back pressure valve opening.

Static output feedback is explored to develop controllers with low complexity. Moreover, the controller design proposed in this paper is pursued with realistic sensor performance limitations in mind. In particular, we show that a static output feedback scheme using anode humidity and various anode side pressure measurements can provide performance comparable to that of a state feedback controller evaluated during various load changes. The static output feedback controller is tuned by optimizing the controller gains for a quadratic cost function [6], [7] similar to the one used for designing the state feedback controller using linear quadratic regulator (LQR) approach. Such a static output feedback control design approach eliminates the need for tuning an observer, as otherwise would be required for implementing the state feedback controller.

We find, however, that one set of output feedback gains is inadequate to address the transient requirements under both subsaturated conditions (only water vapor is present in the stack) as well as saturated cathode conditions (water is present in both vapor and liquid phase inside the cathode compartment). A change in the dynamics of the water mass in the cathode during a saturated cathode condition (such as following a step-up in load) causes a slower return of the cathode water level to its desired condition. In addition, it is difficult to regulate the cathode humidity using anode side measurements due to a weak influence of liquid water in the cathode on the water transport through the membrane. Through analysis of system dynamics, we demonstrate that the performance objectives can be satisfied using a gain-scheduled static output feedback scheme that switches the controller gains when the cathode vapor pressure reaches the saturation pressure for water vapor. Although the discussion in this paper will focus on the cathode humidity-based gain-switching, the controller gains have also been scheduled based upon the stack current to account for other system nonlinearities.

The objective of this study is to first develop a control system for water management using an ejector-based anode recirculation system and then demonstrate the use of controller gain switching to address the strong nonlinearity associated with membrane water transport. The performance of the control system is evaluated through simulations assuming perfect knowledge of the plant dynamics. These dynamics depend upon factors such as the temperature of the fuel cell stack or water transport characteristics. Additional investigation required to evaluate the robustness of the control system to these factors or other plant uncertainties is beyond the scope of this paper.

The paper is organized as follows. In Section II, we describe the system model and then formulate the control problem in Section III. A state feedback controller is developed and implemented in Section IV to obtain a benchmark for the transient response. In Section V, an output feedback controller using pressure and humidity measurements is designed assuming subsaturated conditions in the stack and the response of the closed loop system is compared with the benchmark for various load changes. To satisfy the transient response requirements under saturated cathode conditions, a gain-scheduled output feedback controller is designed. The results are summarized in Section VI.

\section{Dynamic Model of the PEM Fuel Cell With ANODE RECIRCULATION SYSTEM}

A lumped-parameter control-oriented model of a $75-\mathrm{kW}$ PEM fuel cell system (see Fig. 1), developed in [2] and [8], is employed for the control analysis in this paper. The nomenclature used for modeling is described in Table I. This dynamic system model involves ten states

$$
\begin{array}{r}
x=\left[\begin{array}{llllll}
m_{\mathrm{H}_{2}, a} & m_{\mathrm{H}_{2} \mathrm{O}, a} & m_{\mathrm{H}_{2}, r} & m_{\mathrm{H}_{2} \mathrm{O}, r} & m_{\mathrm{H}_{2}, s m, a} \\
m_{\mathrm{O}_{2}, c} & m_{\mathrm{H}_{2} \mathrm{O}, c} & m_{\mathrm{N}_{2}, c} & m_{s m, c} & m_{o m, c}
\end{array}\right]^{T}
\end{array}
$$

where $m_{i, j}$ denotes the mass of species " $i$ " in manifold " $j$." The subscripts " $a$, ," " $r$," "sm, $a$," "c," "sm, $c$," and "om, $c$ " refer to the anode, return manifold, anode supply manifold, cathode, cathode supply manifold, and cathode outlet manifold, respectively. The constituents in these manifolds are denoted by using the subscripts $\mathrm{H}_{2}, \mathrm{H}_{2} \mathrm{O}, \mathrm{O}_{2}$, and $\mathrm{N}_{2}$, which represent hydrogen, water, oxygen, and nitrogen, respectively.

Assuming that the fuel cell system is maintained at a constant temperature, the system dynamics are modeled by applying mass conservation to each species in a given manifold as

$$
\frac{d m_{i, j}}{d t}=W_{i, j}^{\text {in }}-W_{i, j}^{\text {out }}
$$

where the mass flow rates of species " $i$ " entering (or generated) and leaving (or consumed) manifold " $j$ " are denoted by $W_{i, j}^{\text {in }}$ and $W_{i, j}^{\text {out }}$, respectively. The various flows that influence the dynamics of the system are modeled in [2] and [8], and the relevant equations are summarized in Tables II-IV.

The water mass dynamics in the anode and cathode are now elaborated as they are critical to the results presented in this work. 
TABLE I

NOMENCLATURE

\begin{tabular}{|c|c|}
\hline \multicolumn{2}{|c|}{ Variables } \\
\hline$a_{w}$ & Water activity \\
\hline$\tilde{k}$ & Flow coefficient $\left[\mathrm{kg} \mathrm{s}^{-1} \mathrm{~Pa}^{-1}\right]$ \\
\hline$m$ & Mass $[\mathrm{kg}]$ \\
\hline$p$ & Pressure $[\mathrm{Pa}]$ \\
\hline$y$ & Mass fraction \\
\hline$A$ & Area $\left[\mathrm{m}^{-2}\right]$ \\
\hline$I_{s t}$ & Stack current $[\mathrm{Amp}]$ \\
\hline$K$ & Feedback gains \\
\hline $\bar{M}$ & Molecular weight $\left[\mathrm{kg} \mathrm{mole}{ }^{-1}\right]$ \\
\hline$N$ & Molar flux [mole $\mathrm{s}^{-1} \mathrm{~cm}^{-2}$ ] \\
\hline$R$ & Gas constant $\left[\mathrm{J} \mathrm{kg}^{-1} \mathrm{~K}^{-1}\right]$ \\
\hline$T$ & Temperature $[\mathrm{K}]$ \\
\hline$\tilde{V}$ & Volume $\left[\mathrm{m}^{3}\right]$ \\
\hline$W$ & Flow rate $\left[\mathrm{kg} \mathrm{s}^{-1}\right]$ \\
\hline$\gamma$ & Specific heat ratio \\
\hline$\lambda$ & Water content \\
\hline$\phi$ & Relative humidity \\
\hline \multicolumn{2}{|c|}{ Superscripts and Subscripts } \\
\hline$a$ & Anode \\
\hline atm & Ambient condition \\
\hline$b p v$ & Back pressure valve \\
\hline$c$ & Cathode \\
\hline comp & Compressor \\
\hline cond & Condenser \\
\hline$g$ & Dry air \\
\hline gen & Generated \\
\hline in & Inlet \\
\hline$l$ & Liquid phase \\
\hline$m$ & Membrane \\
\hline om & Outlet manifold \\
\hline out & Outlet \\
\hline$r$ & Return manifold \\
\hline rem & Water removed \\
\hline sat & Saturated \\
\hline$s m$ & Supply manifold \\
\hline st & Stack \\
\hline sup & Fuel supply \\
\hline$v$ & Vapor phase \\
\hline $\mathrm{H}_{2}$ & Hydrogen \\
\hline $\mathrm{H}_{2} \mathrm{O}$ & Water \\
\hline $\mathrm{O}_{2}$ & Oxygen \\
\hline $\mathrm{N}_{2}$ & Nitrogen \\
\hline
\end{tabular}

TABLE II

EQUATIONS GOVERNING REACTANT CONSUMPTION ALONG WITH WATER GENERATION AND TRANSPORT INSIDE THE STACK

Electrochemical reactions

(A1) Cathode, Water generated $\quad W_{\mathrm{H}_{2} \mathrm{O}, \text { gen }}=n_{\text {cells }} \frac{I_{s t}}{2 F} \bar{M}_{\mathrm{H}_{2} \mathrm{O}}$

(A2) Cathode, Oxygen consumed $\quad W_{\mathrm{O}_{2}, \text { rct }}=n_{\text {cells }} \frac{I_{s t}}{4 F} \bar{M}_{\mathrm{O}_{2}}$

(A3) Anode, Hydrogen consumed $\quad W_{\mathrm{H}_{2}, r c t}=n_{\text {cells }} \frac{I_{s t}}{2 F} \bar{M}_{\mathrm{H}_{2}}$

Membrane water transport

(B1) Net flow, Anode to Cathode $\quad W_{m e m b}=\bar{M}_{\mathrm{H}_{2} \mathrm{O}} A_{f c} n_{\text {cells }}\left(N_{d r a g}-N_{\text {diff }}\right)$

(B2) Electro-osmotic drag, molar flux $\quad N_{d r a g}=\frac{I_{s t}}{A_{f c} F} n_{d}$

(B3) Back diffusion, molar flux $\quad N_{\text {diff }}=\bar{D}_{w}\left(\lambda_{c}-\lambda_{a}\right)$

\section{A. Stack Water Model}

The water masses in the two compartments are given by [2]

$\frac{\mathrm{d} m_{\mathrm{H}_{2} \mathrm{O}, c}}{\mathrm{~d} t}=W_{\mathrm{H}_{2} \mathrm{O}, \text { gen }}+W_{\mathrm{H}_{2} \mathrm{O}, \text { in }, c}-W_{\mathrm{H}_{2} \mathrm{O}, \text { out }, c}+W_{\text {memb }}$

$\frac{\mathrm{d} m_{\mathrm{H}_{2} \mathrm{O}, a}}{\mathrm{~d} t}=W_{\mathrm{H}_{2} \mathrm{O}, \text { in }, a}-W_{\mathrm{H}_{2} \mathrm{O}, \text { out }, a}-W_{\mathrm{memb}}$

where $W_{\text {memb }}$ is the water transport through the membrane from the anode to the cathode. The water generation rate due

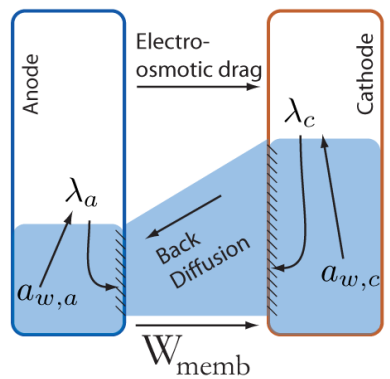

Fig. 2. Water transport between the anode and cathode.

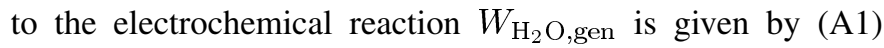
in Table II, where $I_{\text {st }}$ is the stack current. The mass flow rates of water entering and leaving the cathode and anode $W_{\mathrm{H}_{2} \mathrm{O}, \text { in }, c}, W_{\mathrm{H}_{2} \mathrm{O} \text {,out, } c}, W_{\mathrm{H}_{2} \mathrm{O}, \text { in }, a}$, and $W_{\mathrm{H}_{2} \mathrm{O} \text {,out, } a}$, are given by (B2) and (B7) in Table IV and (B3) and (B6) in Table III, respectively.

From the various flows that appear in (3) and (4), the two important flows that depend significantly upon the phase of water inside an electrode compartment are $W_{\text {memb }}$, and the water flowing out of the electrode compartment along with unconsumed reactants. The water transport through the membrane is given by

$$
W_{\text {memb }}=\bar{M}_{\mathrm{H}_{2} \mathrm{O}} A_{\mathrm{fc}} n_{\text {cells }}\left(N_{\text {drag }}-N_{\text {diff }}\right)
$$

where $A_{f c}$ denotes the active fuel cell area and $n_{\text {cells }}$ is the number of cells in the stack. The molar flux per unit cell from the anode to the cathode due to the electro-osmotic drag is $N_{\text {drag }}$, while $N_{\text {diff }}$ is the molar flux per unit cell due to the back diffusion from the cathode to the anode caused by the difference in water concentration on the anode and cathode sides of the membrane. These two flows are shown in Fig. 2. The molar flux due to electro-osmotic drag from anode to cathode $N_{\text {drag }}$ depends upon the load $I_{\text {st }}$ and is given by [9]

$$
N_{\mathrm{drag}}=\frac{I_{\mathrm{st}}}{A_{\mathrm{fc}} F} n_{d}
$$

where $n_{d}$ is the drag coefficient and $F$ is the Faraday's constant. The back diffusion depends upon the difference in the water contents at the electrode membrane interface on the anode and cathode water sides (see Fig. 2). The back diffusion is modeled as [9]

$$
N_{\text {diff }}=\bar{D}_{w}\left(\lambda_{c}-\lambda_{a}\right)
$$

where $\bar{D}_{w}$ is the diffusion coefficient and $\lambda_{c}$ and $\lambda_{a}$ are the cathode and anode water contents, respectively.

The water content at an electrode-membrane interface $\lambda_{j}$ depends upon the water level inside the adjacent electrode compartment " $j$," which is represented using the water activity $a_{w, j}$. The relation between $a_{w, j}$ and $\lambda_{j}$ is shown in Fig. 3, where the 
TABLE III

Governing EQuATIONS For the ANOde Flow PATH SHOWn IN Fig. 1

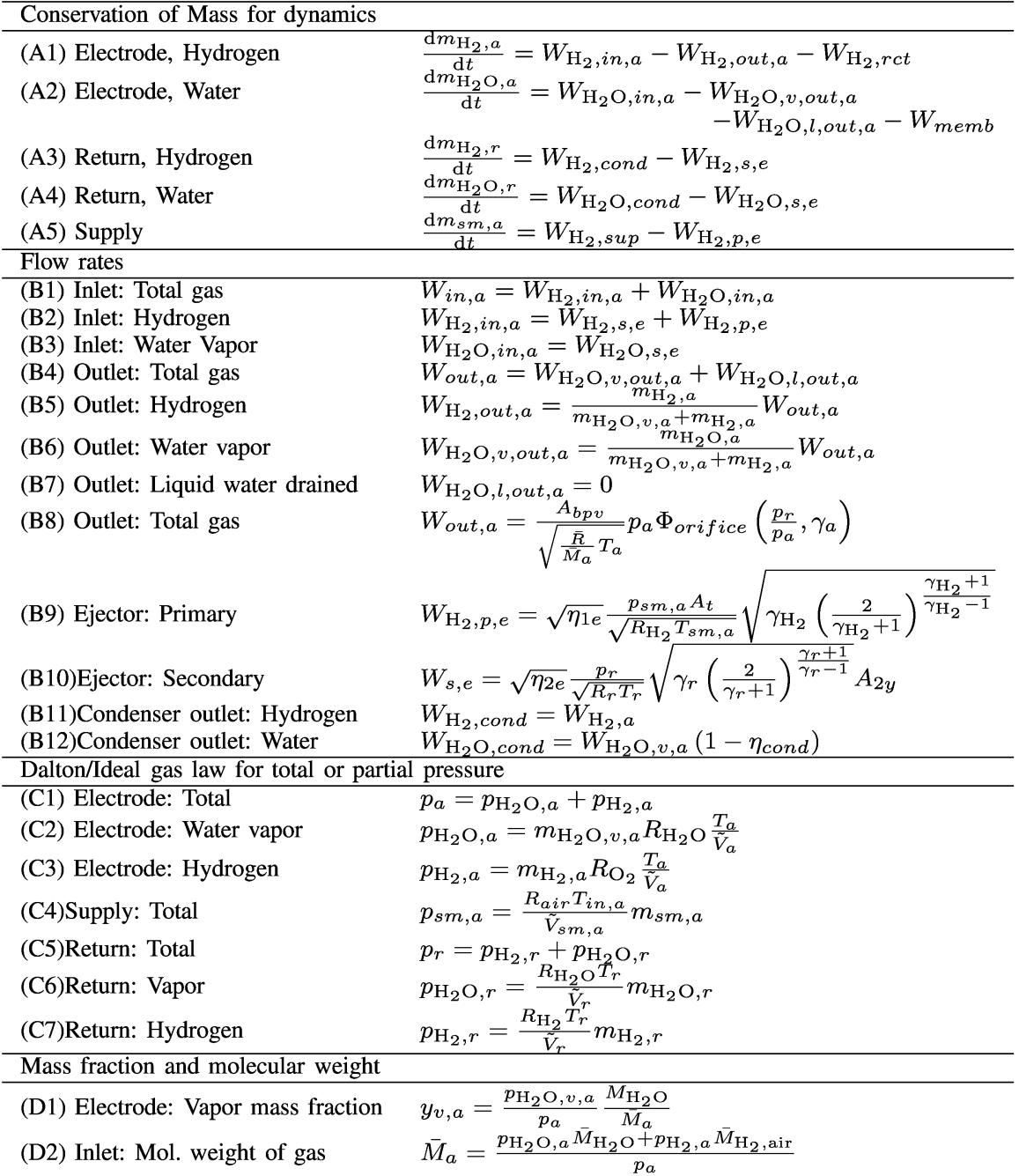

water activity in an electrode compartment is calculated from the mass of water in that compartment using [10]

$$
a_{w, j}=\frac{m_{\mathrm{H}_{2} \mathrm{O}, j}}{m_{\mathrm{H}_{2} \mathrm{O}, v, j}^{\mathrm{sat}_{2}}}
$$

where " $j$ " is replaced by " $a$ " for anode and " $c$ " for cathode.

The maximum mass of water in vapor phase that can be present in an electrode compartment " $j$," $m_{\mathrm{H}_{2} \mathrm{O}, v, j}^{\mathrm{sat}}$, is given by

$$
m_{\mathrm{H}_{2} \mathrm{O}, v, j}^{\mathrm{sat}}=\frac{p_{\mathrm{sat}}\left(T_{j}\right) \tilde{V}_{j}}{R_{\mathrm{H}_{2} \mathrm{O}} T_{j}}
$$

where $p_{\text {sat }}\left(T_{j}\right)$ denotes the saturation pressure corresponding to temperature $T_{j}, \tilde{V}_{j}$ is the lumped volume of the compartment, and $R_{\mathrm{H}_{2} \mathrm{O}}$ is the gas constant of water vapor. Note that, when liquid water is present inside the electrode compartment, that is, $a_{w, j}>1$, the slope of $\lambda_{j}$ versus $a_{w, j}$ is significantly lower, which affects the membrane water transport characteristics.

Another characteristic affected by the phase change of water inside an electrode compartment is $W_{\mathrm{H}_{2} \mathrm{O}, \text { out, } j}$, the flow rate of water leaving the compartment as a part of the exit flow. Assuming evaporation of water as the only mechanism through which water leaves the compartment, ${ }^{1} W_{\mathrm{H}_{2} \mathrm{O}, \text { out }, j}$ is calculated as

$$
W_{\mathrm{H}_{2} \mathrm{O}, \text { out }, j}=y_{v, j} W_{\text {out }, j}
$$

where the mass fraction of water vapor in the compartment is

$$
y_{v, j}=\frac{m_{\mathrm{H}_{2} \mathrm{O}, v, j}}{m_{\mathrm{H}_{2} \mathrm{O}, v, j}+m_{g, j}}
$$

with $m_{g, j}$ representing the total mass of the dry gas inside the compartment. For example, in the cathode, $m_{g, c}=m_{\mathrm{O}_{2}, c}+$ $m_{\mathrm{N}_{2}, c}$. Assuming instantaneous condensation and evaporation of water, the mass of water vapor is given by

$$
m_{\mathrm{H}_{2} \mathrm{O}, v, j}=\min \left\{m_{\mathrm{H}_{2} \mathrm{O}, j}, m_{\mathrm{H}_{2} \mathrm{O}, v, j}^{\mathrm{sat}}\right\}
$$

where $m_{\mathrm{H}_{2} \mathrm{O}, v, j}^{\mathrm{sat}}$ is the maximum water in vapor phase that can be present in the electrode [see (9)].

${ }^{1}$ Due to this assumption, an increase in liquid water mass inside a compartment does not increase the mass flow rate of water flowing out of the compartment. 
TABLE IV

Governing Equations for the CATHode Air Flow Path Shown IN Fig. 1

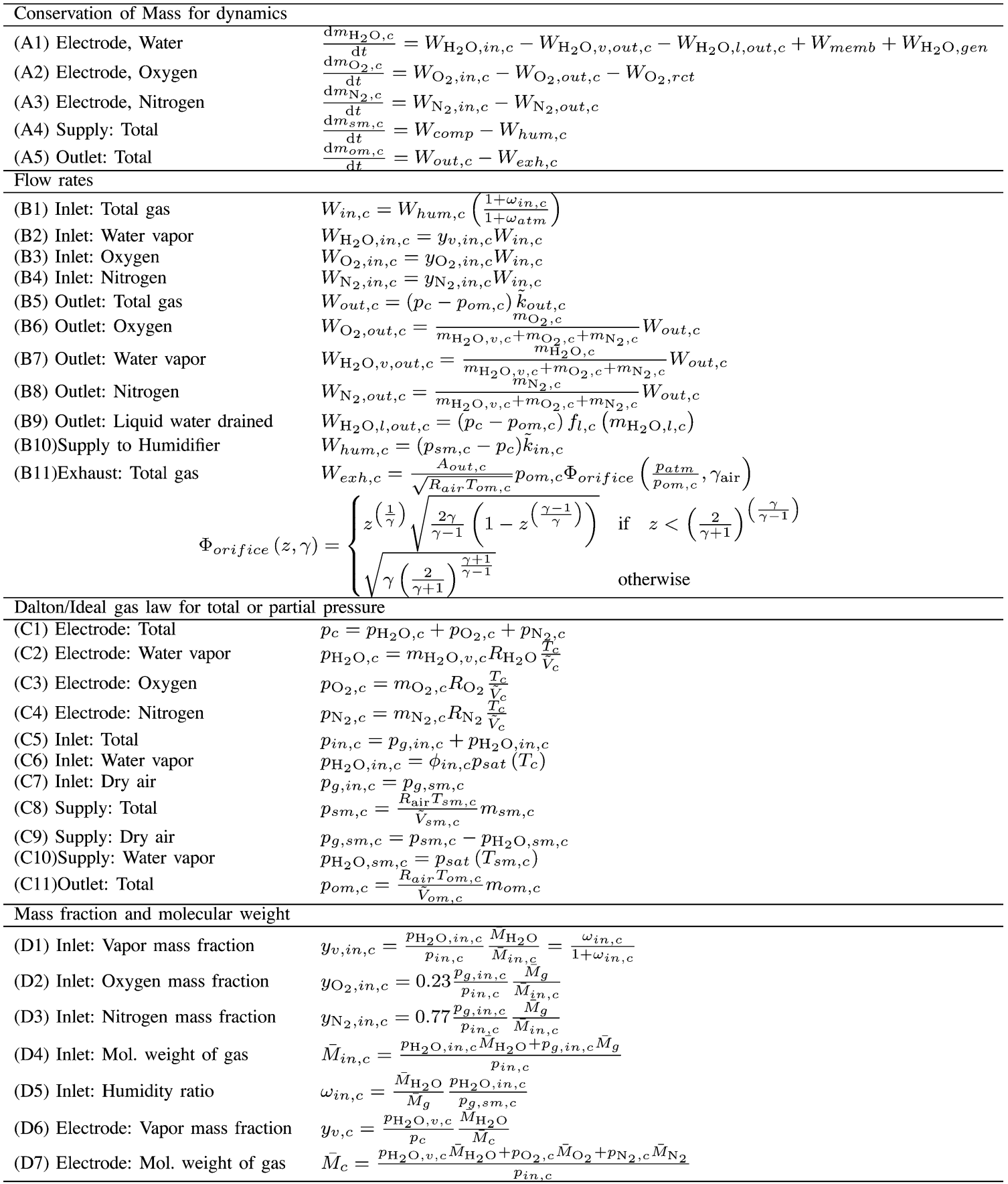

The net mass flow rate of the gas leaving an electrode compartment $W_{\text {out, } j}$ is modeled differently for the cathode and the anode. On the anode side, $W_{\text {out }, a}$ is given by a nonlinear orifice flow equation (see (B8) in Table III) with the area of the orifice $A_{\mathrm{bpv}}$ controlled using the back pressure valve opening $u_{\mathrm{bpv}}$. A nonlinear orifice equation is used to model $W_{\text {out }, a}$, as a significant reduction in the orifice area would result in a large pressure difference across the back pressure valve. Unlike the anode side, the pressure difference between the cathode and the cathode outlet manifold is small, hence, a linear flow equation is adequate to describe $W_{\text {out }, c}$ (see (B5) in Table IV).
B. Control-Oriented Model With Water Activities, Pressures, and Humidities as States

While the system dynamics can be represented using the masses inside the various manifolds and electrode compartments as states, these states cannot be directly measured. For the convenience of state feedback control design and analysis, an alternative system dynamics representation is given here that uses as states the anode and cathode water activities (such that the electrode water mass in both liquid and vapor phases is accounted for), along with various physically measurable variables, such as pressures and humidities. 


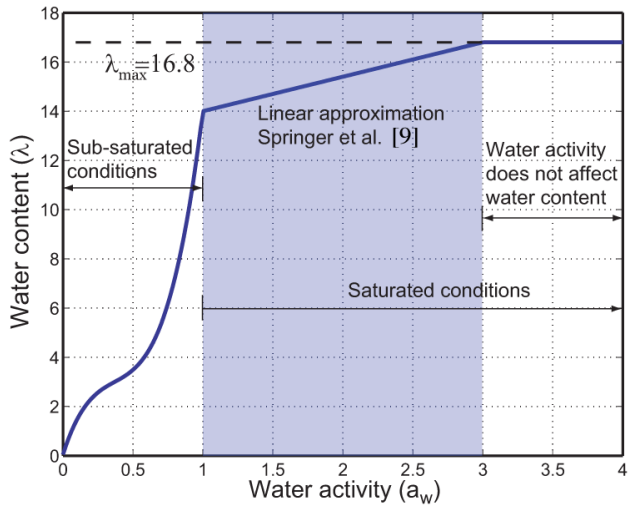

Fig. 3. Water content as a function of water activity.

For manifold " $j$," the relative humidity $\phi_{j}$ is defined as

$$
\phi_{j}=\min \left\{\frac{m_{\mathrm{H}_{2} \mathrm{O}, j}}{m_{\mathrm{H}_{2} \mathrm{O}, v, j}^{\mathrm{at}}}, 1\right\}
$$

where $m_{\mathrm{H}_{2} \mathrm{O}, v, j}^{\mathrm{sat}}$, required in (8), is the mass of water vapor in the manifold at saturation. Note that, for subsaturated cases, $a_{w, j}=\phi_{j}$. The partial pressure of species " $i$ " in manifold " $j$," $p_{i, j}$, and the total pressure in the manifold, $p_{j}$, are given by

$$
\begin{aligned}
p_{i, j} & = \begin{cases}m_{i, j} \frac{R_{i} T_{j}}{\tilde{V}_{j}}, & \text { for } i \neq \mathrm{H}_{2} \mathrm{O} \\
\min \left\{m_{i, j}, m_{\mathrm{H}_{2} \mathrm{O}, v, j}^{\mathrm{sat}}\right\} \frac{R_{i} T_{j}}{\tilde{V}_{j}}, & \text { for } i=\mathrm{H}_{2} \mathrm{O}\end{cases} \\
p_{j} & =\sum_{i} p_{i, j}
\end{aligned}
$$

where $R_{i}$ is the gas constant for species " $i$." Therefore, the system dynamics can also be represented using the states

$$
\begin{array}{rlrllll}
x=\left[\begin{array}{llllll}
p_{a} & a_{w, a} & p_{r} & \phi_{r} & p_{s m, a} \\
& p_{c} & a_{w, c} & p_{\mathrm{O}_{2}, c} & p_{s m, c} & p_{\text {om }, c}
\end{array}\right]^{T} .
\end{array}
$$

The four important modeling assumptions that affect the control analysis presented in this paper are given here.

- A constant and uniform temperature is maintained throughout the fuel cell system by a thermal management system.

- The pressure inside an electrode compartment is not affected by the liquid water inside the compartment.

- The gas flowing out of an electrode compartment does not carry any liquid water.

- The water transport through the PEM can be calculated from the conditions inside the electrode channel instead of having to model the gas diffusion layer separating the channel and the membrane [2].

The first assumption is quite standard for PEMFC control analysis not involving thermal management system, and the temperature can be considered as a slowly varying and measured parameter. The reason for the second assumption is the significantly lower specific volume for liquid water compared with water vapor. To relax the third assumption, substantial investigation is required to understand the interaction between the water inside the electrode compartment and the liquid water removal from the compartment. This is beyond the scope of this paper.

This fuel cell system model is used for the control analysis presented in the following sections. We will begin the analysis by defining the various inputs, the performance variables, and the structure of the controller used in this study.

\section{CONTROL PROBLEM Formulation}

\section{A. Description of Plant Inputs}

For the control problem considered in this study, feedback controllers are developed for the actuators on the anode side of fuel cell stack, therefore the control inputs are defined as

$$
u=\left[\begin{array}{ll}
W_{\text {sup }} & u_{\mathrm{bpv}}
\end{array}\right]^{T}
$$

where $W_{\text {sup }}$ is the flow rate of dry hydrogen supplied from the hydrogen fuel source and $u_{\mathrm{bpv}}$ is the normalized back pressure valve opening. Besides $u$, the other inputs that are relevant to the control problem are referred to as exogenous inputs $w$ defined as

$$
w=\left[\begin{array}{lll}
I_{\mathrm{st}} & \phi_{\mathrm{in}, c} & W_{\mathrm{comp}}
\end{array}\right]^{T}
$$

where $I_{\mathrm{st}}$ is the load, $\phi_{\mathrm{in}, c}$ the cathode inlet humidity, and $W_{\text {comp }}$ the air flow through the compressor.

\section{B. Control Objectives}

The water management requirements for a PEMFC system involve maintaining a water balance inside the stack and achieving desired water activities inside the anode and cathode. The desired water activities for the anode and cathode, $a_{w, a}^{*}$ and $a_{w, c}^{*}$, respectively, are selected such that high humidity conditions (barely below saturation) are maintained inside the two compartments of the fuel cell stack, yet the compartments are subsaturated at equilibrium. The specific choice of $a_{w, a}^{*}$ and $a_{w, c}^{*}$ will be discussed later in Section III-C.

In addition to water management, the pressure difference across the membrane should be regulated to avoid excessive stress on the membrane. For the high-pressure fuel cell system considered in this study, the cathode side pressures vary between $1 \cdot 10^{5}$ and $3 \cdot 10^{5} \mathrm{~Pa}$ for the operating range of the load. Regardless of this variation in cathode pressure, an acceptable pressure difference across the membrane should be maintained throughout the operating range by regulating the anode pressure $p_{a}$ to follow the pressure on the cathode side. Similar to [2], the desired anode pressure $p_{a}^{*}$ is obtained from the cathode supply manifold pressure $p_{\mathrm{sm}, c}$ as

$$
p_{a}^{*}=0.923 p_{\mathrm{sm}, c}+4850
$$

where both $p_{a}^{*}$ and $p_{\mathrm{sm}, c}$ are in Pa. 


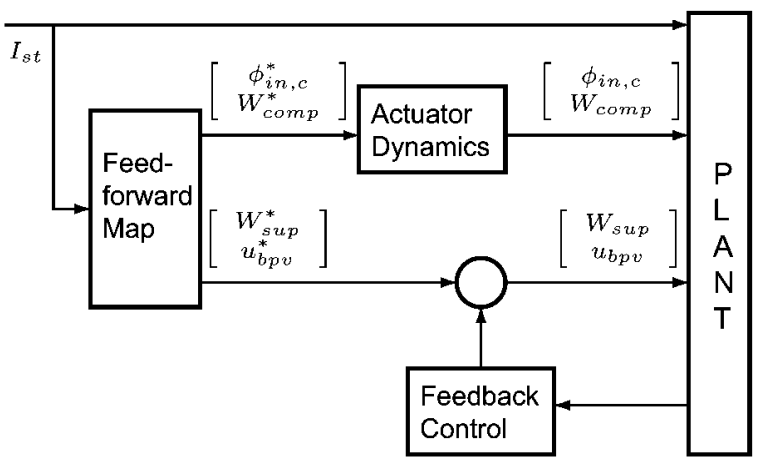

Fig. 4. Controller structure showing the feedforward and feedback control signals.

For subsequent control system development, the pressure regulation and water management objectives of the system are represented using the performance variables $z$ defined as

$$
z=\left[\begin{array}{lll}
a_{w, a}-a_{w, a}^{*} & a_{w, c}-a_{w, c}^{*} & p_{a}-p_{a}^{*}
\end{array}\right]^{T}
$$

where $a_{w, a}, a_{w, c}$, and $p_{a}$ are the anode water activity, the cathode water activity, and the anode pressure, respectively. These performance variables deviate from $z=0$ with a load change that: 1) disturbs the pressures and water activities inside the electrodes and 2) changes their desired steady-state values. Minimizing the deviations in $z$ during the load transients and restoring $z$ to the desired equilibrium $(z=0)$ will be accomplished by the control system through proper actuation of $u$.

In general, the controller should be designed to minimize errors in the performance variables, without excessive use of the control inputs. The quantitative transient requirements for the performance variables should be determined by the membrane properties and the construction of the fuel cell stack. Based upon our existing search in the open literature, such specifications are not currently available. Instead, the following transient performance specifications, evaluated using a series of step changes in the load, are adopted to demonstrate the controller design methodology presented in this study.

- The settling time, defined as the time required for the system to settle within an error less than $5 \%$ of its peak error after a load step change, should be less than $3 \mathrm{~s}$ for the anode pressure regulation.

- The settling time should be less than $4 \mathrm{~s}$ for the anode and cathode water activity regulation.

The need for a faster system response would impose more stringent specifications on the settling times.

These transient response requirements are satisfied using a combined static feedforward and feedback controller structure, as shown in Fig. 4. The feedforward map provides the steadystate actuator settings to meet the water management and the fuel supply requirements corresponding to the load. The feedback controller then facilitates the regulation of the performance variables to assure the desired transient response.

\section{Actuator Settings Using Load-Based Feedforward Control}

The feedforward commands are denoted using the superscript "*” in Fig. 4. The maps that provide the feedforward commands
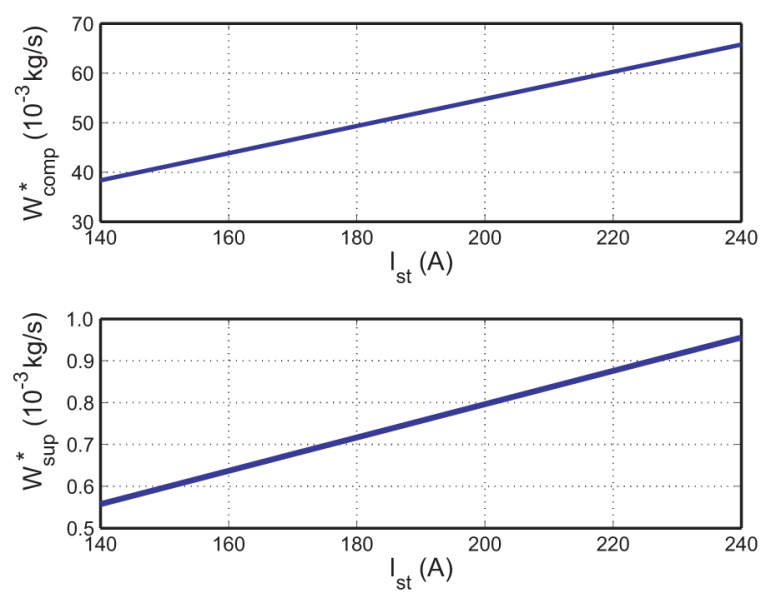

Fig. 5. Steady-state maps of compressor flow and fuel supply for different stack currents.

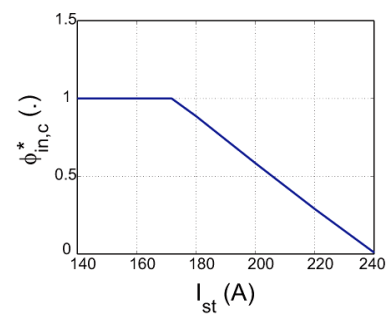

(a)

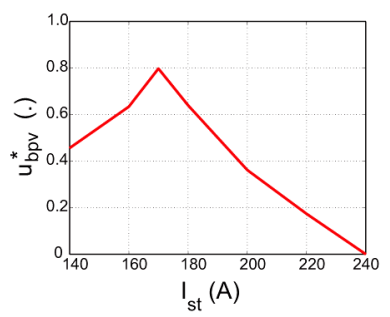

(b)

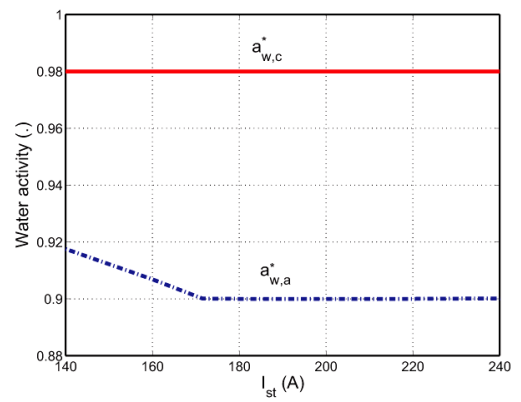

(c)

Fig. 6. Plots (a) and (b) show the desired back pressure valve opening and shows the cathode inlet humidification with respect to the stack current for $a_{w, c}=0.98$. The resulting anode water activity (dashed-dotted line) is shown in (c).

for the cathode air supply from compressor and the anode fuel supply, $W_{\text {comp }}^{*}$ and $W_{\text {sup }}^{*}$, respectively, are shown in Fig. 5, where $W_{\text {comp }}^{*}$ increases with $I_{\text {st }}$ to maintain the oxygen excess ratio at $2[2]$, whereas $W_{\text {sup }}^{*}$ increases to replenish the fuel consumed inside the stack.

While the maps shown in Fig. 5 are independent of the water management requirements, the values for desired cathode inlet humidity and back pressure valve opening, $\phi_{\mathrm{in}, c}^{*}$ and $u_{\mathrm{bpv}}^{*}$, respectively, depend upon the choice of water management scheme. In this study, $\phi_{\text {in, }}^{*}$ varies with $I_{\text {st }}$ according to the function given in Fig. 6(a). This choice of $\phi_{\mathrm{in}, c}^{*}$ then dictates the anode water removal requirement. Fig. 6(b) shows the back pressure valve opening that provides the recirculation flow necessary to meet this requirement [10]. Note that, between loads of 140 to $170 \mathrm{~A}, \phi_{\mathrm{in}, c}^{*}$ is fixed, and hence the anode water removal increases with the load to compensate for the increase 


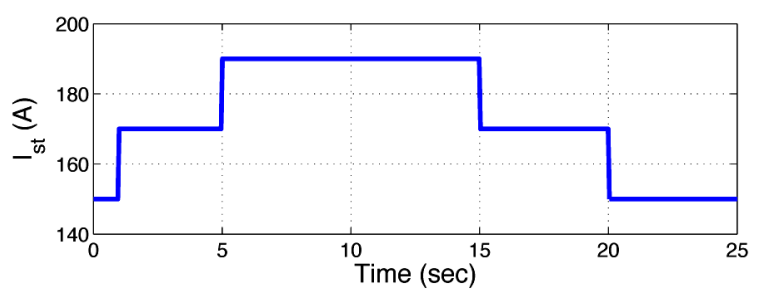

Fig. 7. Current demand profile used to evaluate the closed-loop response.

in the water generation rate. The higher anode water removal is met by using a larger back pressure valve opening.

The decrease in $\phi_{\mathrm{in}, c}^{*}$ with load for $I_{\mathrm{st}}>170 \mathrm{~A}$ is necessitated by the compounded effects of: 1) increased water generation and 2) the limitation in anode water removal. As the water is generated at the cathode, an increase in anode water removal requires a higher back diffusion, and hence a higher activity gradient from the cathode to the anode. For a fixed $a_{w, c}$, a higher gradient is achieved by lowering $a_{w, a}$. Given a requirement that the steady state $a_{w, a}$ should be greater than 0.9 , the anode water removal is constrained by the limitation on back diffusion. Specifically, for $a_{w, a}>0.9$, when the current exceeds $170 \mathrm{~A}$, the water balance can no longer be achieved by keeping $\phi_{\mathrm{in}, c}^{*}$ constant at 1 and manipulating $u_{\mathrm{bpv}}^{*}$ to increase anode water removal. Therefore, the cathode inlet humidity is decreased as the load increases, which leads to a reduction in the anode water removal requirement and consequently a decrease in desired back pressure valve opening. As seen in Fig. 6(c), the desired anode water activity is above 0.9 with $a_{w, c}^{*}=0.98$ when using the $\phi_{\text {in.c }}^{*}$ as shown in Fig. 6(a).

Although the commands for the exogenous inputs are scheduled with the load, $W_{\text {comp }}$ and $\phi_{\text {in, } c}$ are affected by actuator dynamics, as indicated in Fig. 4. Specifically, the dynamics of $\phi_{\text {in, } c}$ are due to the slow response time of the humidifiers [11], and the dynamics of $W_{\text {comp }}$ result from the inertia of the compressor-motor shaft [2]. The specific load profile chosen for controller evaluation is shown in Fig. 7, while Fig. 8 shows the corresponding profiles for the exogenous inputs corresponding to this load profile. This load profile is selected, specifically, to evaluate transient operation scenarios with and without changes in cathode inlet humidity, as seen in Fig. 8(b).

\section{Feedback Controller}

A unique complexity arising from the use of ejector-based recirculation is the need for feedback controller, even if an accurate feed forward map is available. The operating characteristics of the ejector based recirculation are such that the anode pressure lacks a self-stabilizing mechanism. The lack of a self-stabilizing mechanism can be shown by applying mass conservation for hydrogen in the closed path formed by the anode recirculation loop [12], [8] and then observing that the hydrogen flows crossing the boundaries of the closed path are independent of the pressures inside the anode recirculation loop.

These hydrogen flows affect the dynamics of the partial pressure of hydrogen in the anode, and hence the anode pressure, and depend upon the load or the fuel supplied. Therefore, the open-loop dynamics of $p_{a}$ from the load as well as from the fuel supplied are Type 1 (that is, a pure integrator in the dynamics of anode pressure from these inputs [12].) In Fig. 9, the

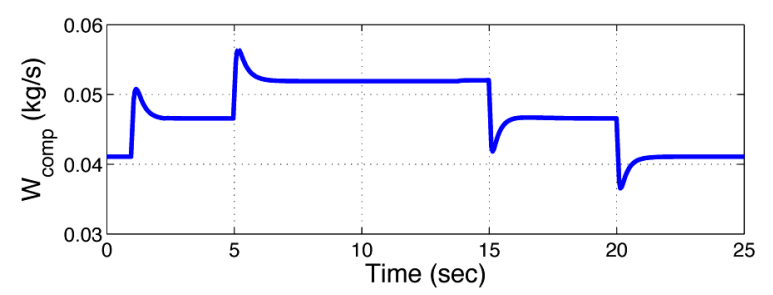

(a)

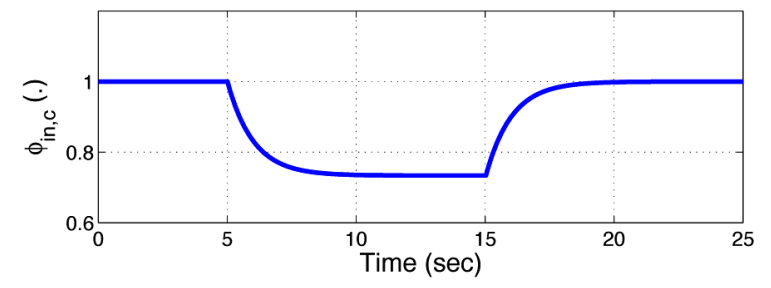

(b)

Fig. 8. The profiles for (a) compressor air supply flow rate and (b) cathode inlet humidification corresponding to the steps in the stack current shown in Fig. 7.

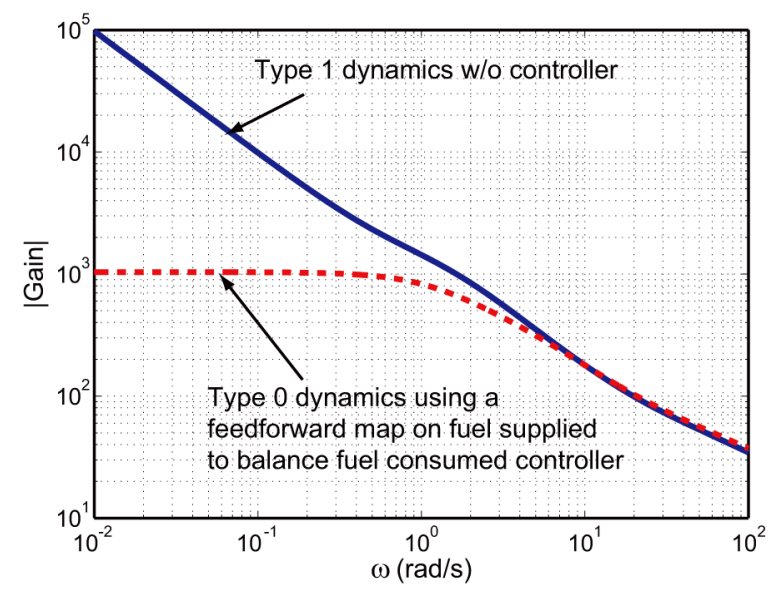

Fig. 9. Bode gain of the transfer function from load $I_{\text {st }}$ to the error in anode pressure $p_{a}-p_{a}^{*}$.

open-loop dynamics of the performance variable $p_{a}-p_{a}^{*}$ from $I_{\text {st }}$ are shown by the black line. The system is not BIBO stable with $I_{\text {st }}$ as the input and $p_{a}-p_{a}^{*}$ as the output, hence, a feedback controller is necessary to provide the stabilizing mechanism.

When using a static feedforward map to coordinate the fuel supply with the fuel consumed, the open-loop dynamics of $p_{a}-$ $p_{a}^{*}$ from the load are Type 0 , as seen from the dashed line in Fig. 9. A Type-0 disturbance can be rejected using a static feedback when the dynamics from the control input are Type 1 [13], hence the pressure set-point tracking to reject disturbance from $I_{\text {st }}$ can be achieved using a static feedback controller combined with a static feedforward map.

While the feedback is critical for tracking anode pressure, the steady anode and cathode humidities can be achieved using an appropriate feedforward control on $u_{\mathrm{bpv}}$. The purpose of using feedback controller for the anode and cathode humidities is to speed up their open-loop dynamics under various operating scenarios.

In this paper, we develop a static feedback controller using pressure and humidity measurements. The pressure measurements are fast (response is of the order of milliseconds), 


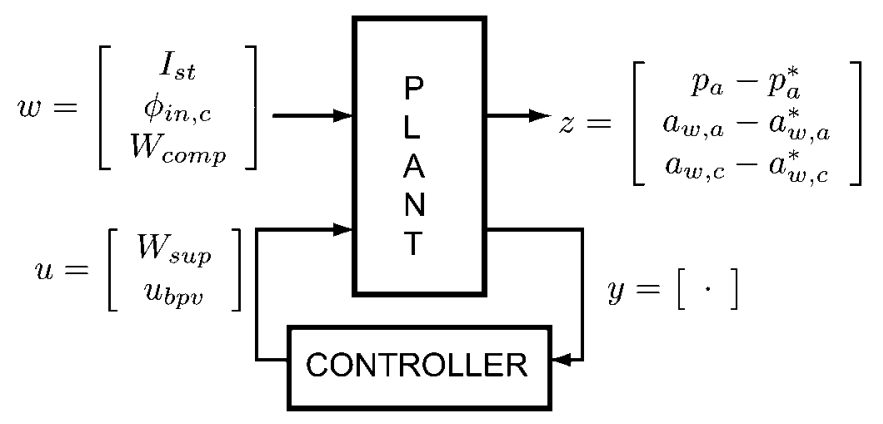

Fig. 10. Standard control problem formulation with $u, w, y$, and $z$ as control inputs, exogenous inputs, measured variables, and performance variables. The choice of $y$ depends upon the type of controller used.

whereas the state-of-the-art humidity measurements are slow (response is of the order of seconds) [14]. As mentioned earlier, for the modularity of control design for the anode recirculation system, we use only anode side humidity and pressure measurements for feedback, therefore, the measured variables are $y=\left[\begin{array}{llll}p_{a} & p_{r} & p_{\mathrm{sm}, a} & \phi_{a}\end{array}\right]$, where $p_{r}$ and $p_{\mathrm{sm}, a}$ are the pressures in the anode return manifold and the anode supply manifold shown in Fig. 1.

The control problem is summarized in Fig. 10 using the standard control notations. The capability of the system to meet the time-domain performance requirements with an appropriate controller is demonstrated in Section IV using a full-state feedback controller, that is, $y=x$. The closed-loop response with a full state feedback controller is then regarded as a benchmark and is used to compare the performance of the control system developed in Section V using $y=\left[\begin{array}{llll}p_{a} & p_{r} & p_{\mathrm{sm}, a} & \phi_{a}\end{array}\right]$ as the measurements.

\section{BENCHMARK CONTROL DESIGN AND PERFORMANCE EVALUATION}

\section{A. Controller Design}

Following the structure shown in Fig. 4, a state feedback controller is combined with a feedforward map such that the control action is given by

$$
u=u^{*}+K_{s f}\left(x-x^{*}\right)
$$

where $x$ is the vector of states in the system, $x^{*}$ represents the desired steady-state operating condition, and $u^{*}=\left[\begin{array}{ll}W_{\mathrm{sup}}^{*} & u_{\mathrm{bpv}}^{*}\end{array}\right]^{T}$ represents the static feedforward commands. The state feedback gains are denoted using $K_{s f}$.

A controller design methodology that directly addresses the performance requirements in time-domain is limited to numerical constrained optimization or trial-and-error tuning of $K_{s f}$. In this study, we take the following indirect yet standard approach. The performance metric is chosen to be the quadratic cost function, as

$$
J=\int_{0}^{\infty}\left(z^{T} Q z+\left(u-u^{*}\right)^{T} R\left(u-u^{*}\right)\right) d t
$$

where $Q$ is a $3 \times 3$ diagonal matrix and $R$ is a $2 \times 2$ diagonal matrix. Here, the matrices $Q$ and $R$ are chosen such that an optimal full-state feedback controller, obtained using the linear quadratic regulator approach for the cost function $J$, satisfies the desired transient criteria for the load profile shown in Fig. 7.

For the controller design, we linearize the system dynamics about an equilibrium condition. Let the linear representation of the system be given by

$$
\begin{aligned}
\delta \dot{x} & =A \delta x+B_{u} \delta u+B_{w} \delta w \\
\delta z & =C_{z} \delta x
\end{aligned}
$$

where $\delta(\cdot)$ denotes the deviation of the variable $(\cdot)$ from the equilibrium condition, for example, $\delta x=x-x^{*}$. The matrices $A, B_{u}, B_{w}$, and $C_{z}$ of the linearized system are obtained through symbolic linearization using MATLAB. Then, the optimal state feedback controller gains that minimize the cost function $J$ are given by [13]

$$
K_{s f}=-R^{-1} B_{u}^{T} \bar{P}
$$

where $\bar{P}$ is a solution to the Algebraic Riccati Equation

$$
A \bar{P}+\bar{P} A+C_{z}^{T} Q C_{z}-\bar{P} B_{u} R^{-1} B_{u}^{T} \bar{P}=0 .
$$

The optimal state feedback controller gains, $K_{s f}$, are scheduled with the load. Namely, the gains are obtained at different loads, by first linearizing the system at the corresponding equilibrium conditions $\left(x^{*}, w^{*}, u^{*}\right)$, and then solving (25) to obtain the expression for $K_{s f}$ using (24). Here, $w^{*}=\left[\begin{array}{lll}I_{\mathrm{st}}^{*} & \phi_{\mathrm{in}, c}^{*} & W_{\mathrm{comp}}^{*}\end{array}\right]^{T}$, such that $\phi_{\mathrm{in}, c}^{*}$ and $W_{\text {comp }}^{*}$ are the steady-state cathode inlet humidity and compressor flow rate corresponding to the load $I_{\mathrm{st}}^{*}$.

The diagonal entries of $Q$ and $R$ were tuned by trial and error, such that the closed-loop response of the state feedback controller satisfied the design criteria. We use the same values for $Q$ and $R$ to obtain $K_{s f}$ at all operating conditions, as only a marginal change in the closed-loop performance is achieved by retuning $Q$ and $R$ at different operating conditions where the controller was evaluated. ${ }^{2}$ In this study, the feedback gains are obtained for

$$
\begin{aligned}
& Q=\left[\begin{array}{ccc}
20 & 0 & 0 \\
0 & 0.15 & 0 \\
0 & 0 & 0.15
\end{array}\right] \\
& R=\left[\begin{array}{cc}
32 & 0 \\
0 & 60
\end{array}\right] .
\end{aligned}
$$

\section{B. Performance Evaluation With a State Feedback Controller}

The performance of the full-state feedback controller is analyzed through simulations for the load profile given in Fig. 7. The closed-loop response of the full-state feedback controller to this stack current profile is shown in Fig. 11 by the solid-black line (SFB w/ $a_{w}$ ). The other lines shown in the same figure correspond to responses with different controllers that will be explained later. A step increase in the stack current causes an initial buildup of water in the cathode, a part of which is transferred to the anode through the membrane. As shown by the transients for the step change in load from 170 to 190 A at time $t=5 \mathrm{~s}$, the dynamics in the cathode humidifier further compounds to

\footnotetext{
${ }^{2}$ The $Q$ and $R$ should be tuned separately for an operating condition when the closed-loop performances do not meet the transient performance requirements.
} 

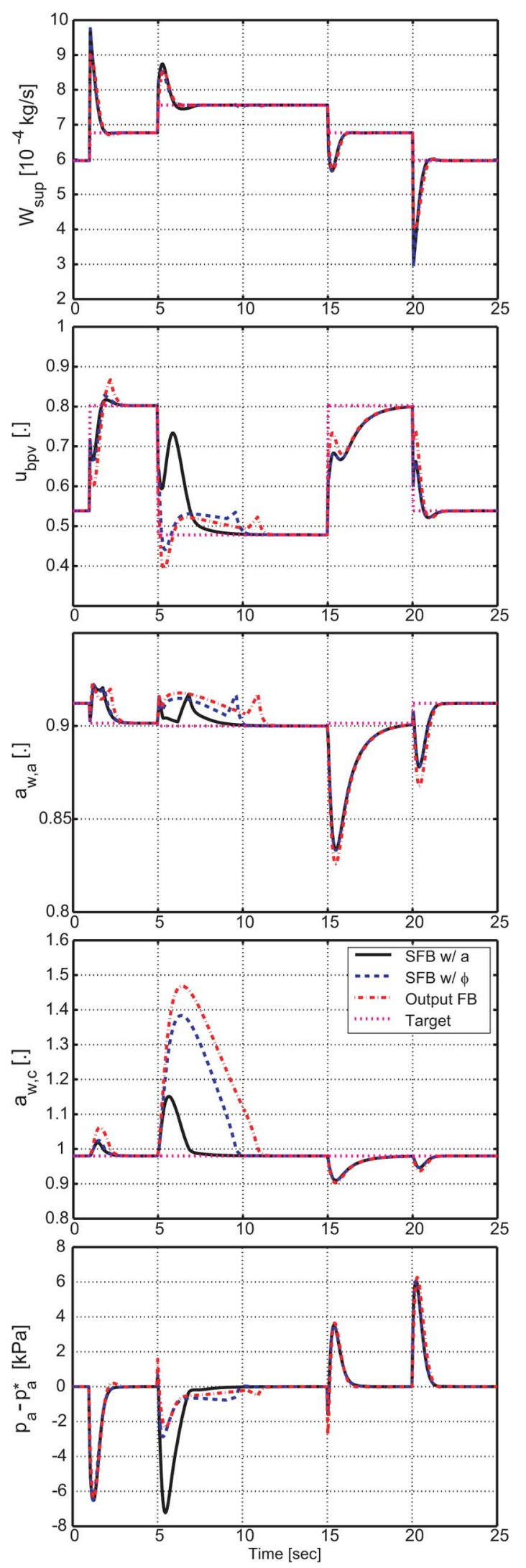

Fig. 11. Responses of state feedback controller using activity measurements, (SFB w/ $a_{w}$ ), humidity measurements (SFB w/ $\phi$ ), and output feedback controller $(\mathrm{OFB})$.

the liquid water accumulation. Although the transient requirements, specified in Section III-B, are satisfied in the presence of this liquid water in the cathode, the responses of $a_{w, a}$ and $a_{w, c}$ are different than that seen during the transients for a load step down. This difference is due to the fact that the presence of liquid water in the cathode $\left(a_{w, c}>1\right)$ causes the following two changes in the water dynamics. First, with the cathode humidity $\phi_{c}=1, a_{w, c}$ does not affect cathode pressure $p_{c}$ or the cathode water vapor mass fraction $y_{v, c}$, as they depend upon $\phi_{c}$. This changes the interaction between $a_{w, c}$ and the flows in/out of the cathode. Second, the change in slope between the water content and the water activity (see Fig. 3) affects the membrane water transport characteristics [9], [15]. In spite of these changes in plant dynamics, the state feedback controller designed assuming subsaturated conditions inside the cathode provides a satisfactory transient response.

Although the responses with the combined full-state feedback and static feedforward controller deliver the desired performance, as shown in Fig. 11, such a controller is difficult to implement due to the following limitations:

- It requires information on each of the ten states while some of the states cannot be measured.

- Using an observer to estimate certain unmeasured states is difficult due to the high order of the plant and complicated nonlinearities associated with membrane water transport.

One particular challenge in implementing the state-feedback controller is the need to measure the cathode water activity. This measurement is limited by the practical difficulties in estimating the amount of liquid water in the cathode compartment. To show the importance of the cathode water activity measurement, the performance of the state feedback controller is evaluated when the cathode water activity measurement is replaced by the cathode humidity measurement. The rest of the states are still assumed to be measurable. The resulting response is shown by the dashed line (SFB w/ $\phi$ ) in Fig. 11. As long as the cathode is sub-saturated, the state feedback controller using $\phi_{c}$ measurement provides the same results as when using $a_{w, c}$ measurement. With liquid water in the cathode $\left(a_{w, c}>1\right)$, however, the use of the humidity sensor in the cathode leads to an error in deducing the cathode water activity. This error deteriorates the response of water activities and increases the settling time for $p_{a}$ as seen in Fig. 11 .

Note that during the transients shown in Fig. 11, the anode humidity sensor is operational, as the anode remains subsaturated $\left(\phi_{a}<1\right)$ even when the cathode is saturated $\left(a_{w, c}>1\right)$. This observation motivates us to investigate the use of the sensor combination $y=\left[\begin{array}{llll}p_{a} & p_{r} & p_{\mathrm{sm}, a} & \phi_{a}\end{array}\right]^{T}$ with a static output feedback controller, aimed at providing a response comparable to the state feedback control but with a substantially reduced number of measurements.

\section{Static Output FeEdBack Controller}

\section{A. Controller Design}

The control action of the combined static output feedback and feedforward controller is given by

$$
u=u^{*}+K_{y}\left(y-y^{*}\right)
$$

where $y^{*}$ are the desired steady-state values of the measured variables and $u^{*}$ represents the feedforward command as de- 
scribed in Section IV.A. The output feedback gains are denoted using $K_{y}$.

Instead of tuning the output feedback controller gains using a trial-and-error approach to meet the performance requirements, we optimize the controller for the performance metric $J$, defined in (22). The optimal feedback gains for the quadratic cost function $J$ are computed by applying an iterative procedure suggested in [6], [7] for a linearized system.

Let the linear representation of the measured variables be given by

$$
\delta y=C_{y} \delta x
$$

where $\delta y$ denotes the deviation of $y$ from its equilibrium condition, $y^{*}$. For the state space representation given by (23) and (28), the optimal feedback gain $K_{y}$, which minimizes the cost function $J$, is then given by [6]

$$
K_{y}=-R^{-1} B_{u}^{T} S L C_{y}^{T}\left(C_{y} L C_{y}^{T}\right)^{-1}
$$

where the matrices $S$ and $L$ are obtained by solving the following coupled Riccati equations [6]:

$$
\begin{aligned}
A_{c l}^{T} S+S A_{c l}+C_{z}^{T} Q C_{z}+C_{y}^{T} K_{y}^{T} R K_{y} C_{y} & =0 \\
A_{c l} L+L A_{c l}^{T}+I & =0
\end{aligned}
$$

with

$$
A_{c l}=A+B_{u} K_{y} C_{y} .
$$

The values for $Q$ and $R$ are chosen as given in (26), such that an optimal full-state feedback controller, obtained using the linear quadratic regulator approach for the cost function $J$, satisfies the desired transient criteria. Similar to the state feedback controller, a gain-scheduled output feedback controller is developed by computing the optimal controller gains $K_{y}$ at various stack currents.

\section{B. Performance Analysis for Subsaturated Conditions}

The response of the closed-loop system with the sensor selection $y=\left[\begin{array}{llll}p_{a} & p_{r} & p_{\mathrm{sm}, a} & \phi_{a}\end{array}\right]^{T}$ and the static output feedback is shown in Fig. 11 by the dashed-dot line (OFB). The feedback gains calculated for subsaturated conditions using $Q$ and $R$ given in (26), are shown by the line with square markers for various operating conditions in Fig. 13. The controller gains are scheduled based upon the fuel cell load. The inputs and outputs are properly scaled to provide sensible comparison of the controller gains.

Several observations which offer insights on the design and implementation are discussed through the following remarks.

Remark I: One can see from Fig. 11 that, when the cathode is subsaturated, the response with output feedback controller is comparable to the benchmark (state feedback) controller. The close matching between the responses for both the controllers under subsaturated conditions can be predicted through linear analysis. Assuming ideal actuators for the cathode inlet humidity and compressor flow, the closed loop sensitivity function of the performance variables to the changes in load can be calculated for the state feedback (SFB) and the output
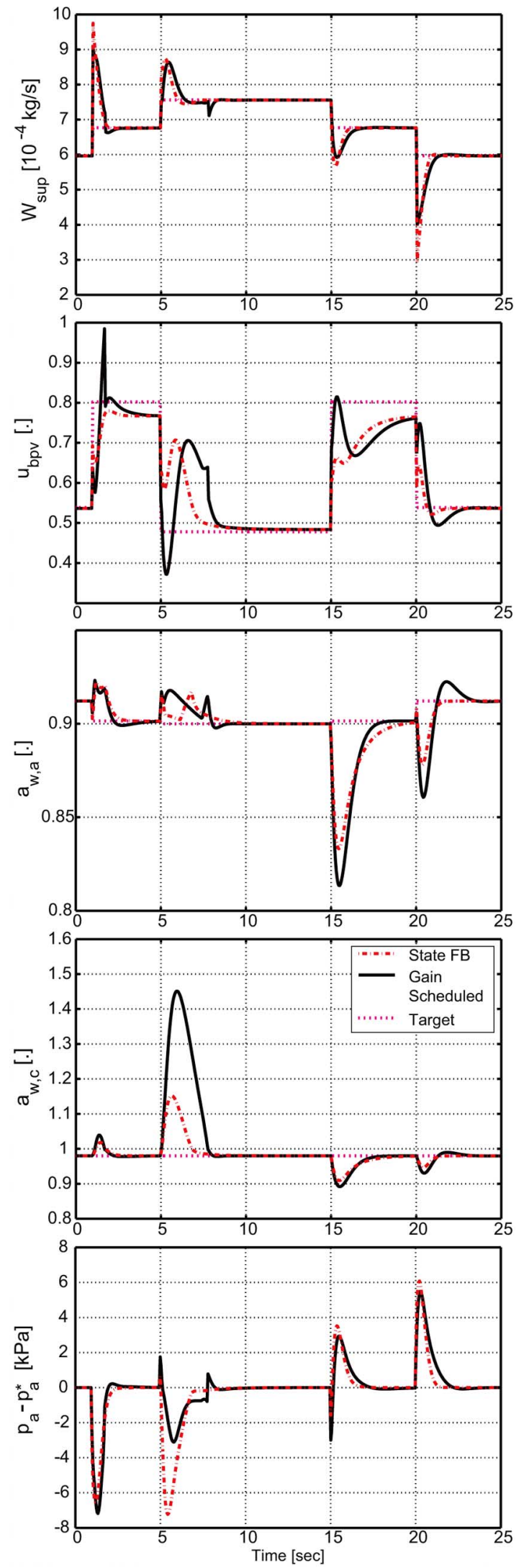

Fig. 12. Comparison of responses of gain-scheduled output feedback controller and the state feedback controllers. The anode humidity sensor used by the gainscheduled controller has a time constant of $1 \mathrm{~s}$. 


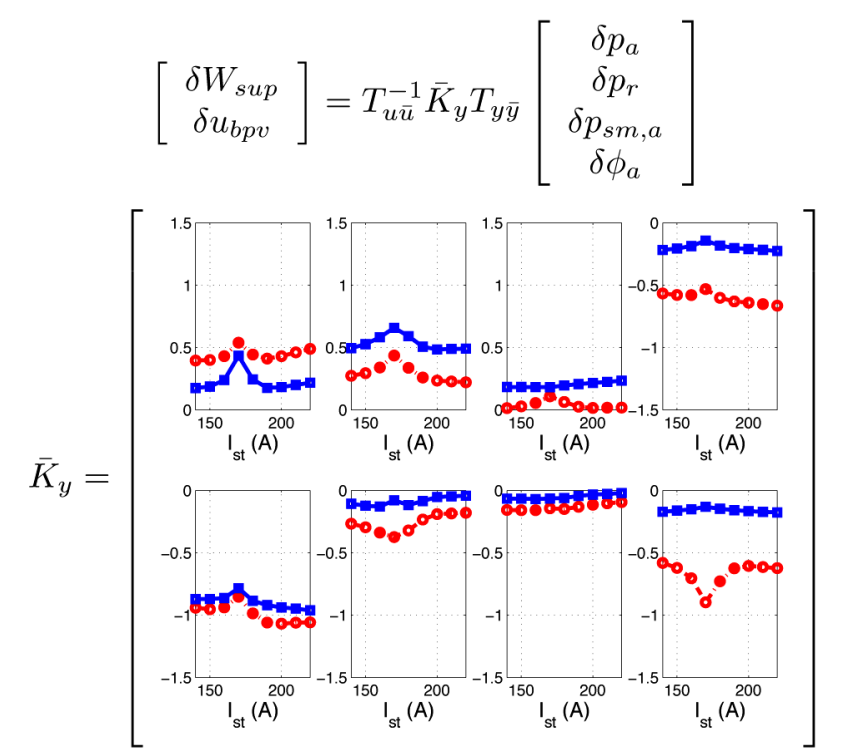

Fig. 13. Gains designed for plants with saturated cathode (circular markers) and subsaturated cathode (square markers). $T_{u \bar{u}}$ and $T_{y \bar{y}}$ are the matrices used to scale the inputs and measurements, respectively.

feedback (OFB) controllers. In Fig. 14, the Bode gain plots of these sensitivity functions are given for both saturated as well as subsaturated cathode conditions. Note that, for subsaturated conditions the responses with state feedback and output feedback controllers are similar as observed from the step response to changes in load. Thus, a static output feedback controller with $y=\left[\begin{array}{llll}p_{a} & p_{r} & p_{\mathrm{sm}, a} & \phi_{a}\end{array}\right]^{T}$ suffices to provide a response comparable with that using a humidity sensor-based full-state feedback.

To verify that the static output feedback is indeed sufficient, we compute a reduced order model by assuming that the dynamics of the unmeasured states are fast compared to the dynamics of the measured states given by $y$. A state feedback controller is then designed for the reduced order plant such that the feedback gains are optimal for the quadratic cost $J$ given in (22). The closed loop sensitivities obtained when applying this controller to the full order plant are similar to the sensitivities when using a full-state feedback controller designed in Section IV for the full order plant model.

Remark II: The output feedback controller designed for a subsaturated cathode condition cannot meet the performance requirements when the cathode is saturated. Substantial increases in settling times for the water activities and a large deviation in cathode water activity can be seen from Fig. 11. While the dynamics of the cathode inlet humidifier lead to excessive liquid water accumulation in the cathode, it is the change in the plant dynamics in the presence of liquid water that causes the significantly slow recovery of the cathode water activity with the static output feedback. It is noteworthy to point out in Fig. 14 that although the sensitivity of $a_{w, c}-a_{w, c}^{*}$ increases with liquid water in the cathode for the state feedback as well as the output feedback controller, this increase in the sensitivity is significantly higher with the static output feedback controller (shown by the dashed line).

This degradation in the response observed in Fig. 14 indicates that an output feedback controller designed for sub-saturated conditions is ineffective when the dynamics of $a_{w, c}$ and $a_{w, a}$ are

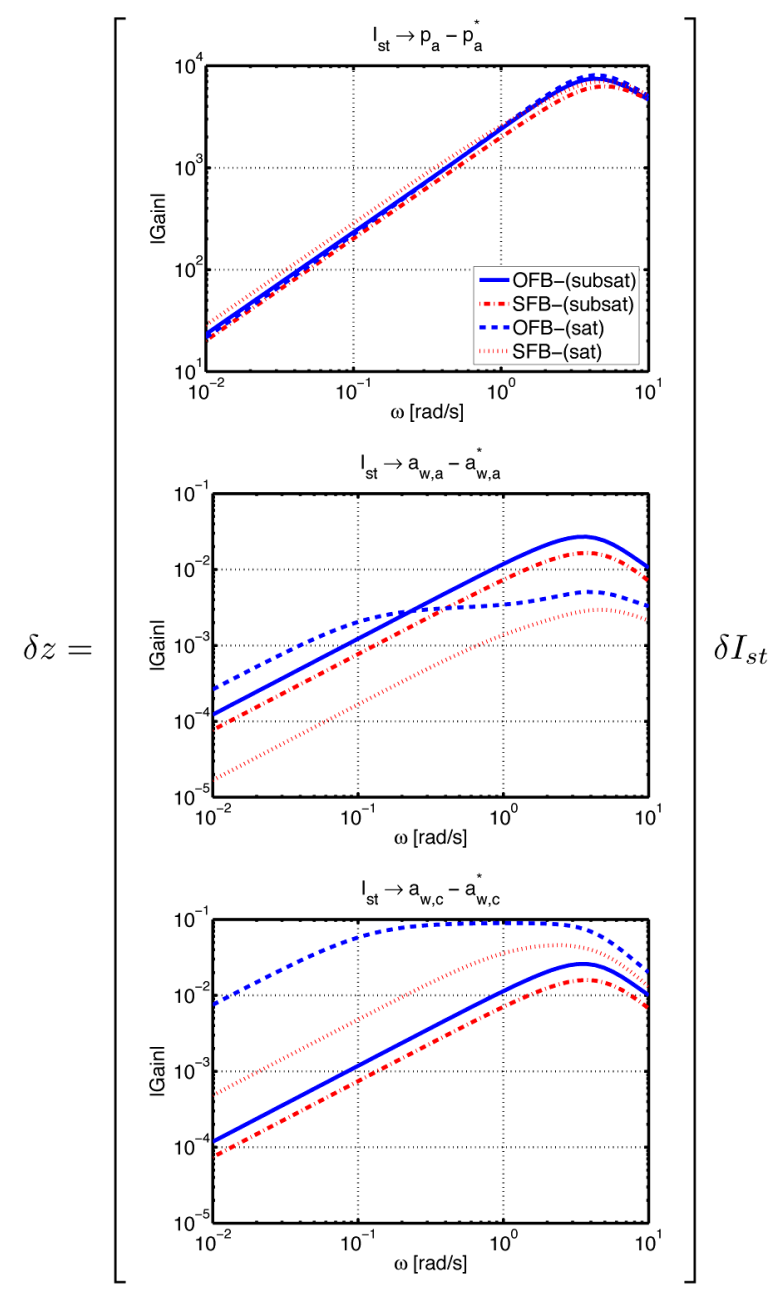

Fig. 14. Closed-loop sensitivity of performance variables to change in load. Controller gains tuned for subsaturated anode and cathode and evaluated for saturated and subsaturated cathode conditions.

altered by the presence of liquid water in the cathode. Further investigation is directed towards understanding this change in plant dynamics, followed by redesigning the output feedback controller under a saturated cathode condition to improve the closed loop performance.

\section{Analysis of Plant Dynamics in Saturated Conditions}

As the $a_{w, c}$ measurement is not used for the output feedback controller, the difficulty in regulating $a_{w, c}$ in the saturated condition could be attributed to poor observability of $a_{w, c}$ from anode side measurements. The interaction between the anode humidity and cathode water activity is investigated by evaluating their closed-loop sensitivities to a disturbance from $\phi_{\mathrm{in}, c}$, as the anode humidity is affected by $\phi_{\mathrm{in}, c}$ only through a change in $a_{w, c}$.

Fig. 15 compares the sensitivity functions of the two closed-loop systems, one with the output feedback controller designed in Section V-B (i.e., for subsaturated conditions), and the other with the state feedback controller. Fig. 15(a) indicates that, when the cathode is subsaturated, both $a_{w, c}$ and $a_{w, a}$ have approximately the same sensitivity to the disturbance. Furthermore, these sensitivities are equal to the sensitivities using the full state feedback controller (not shown in the plot for 


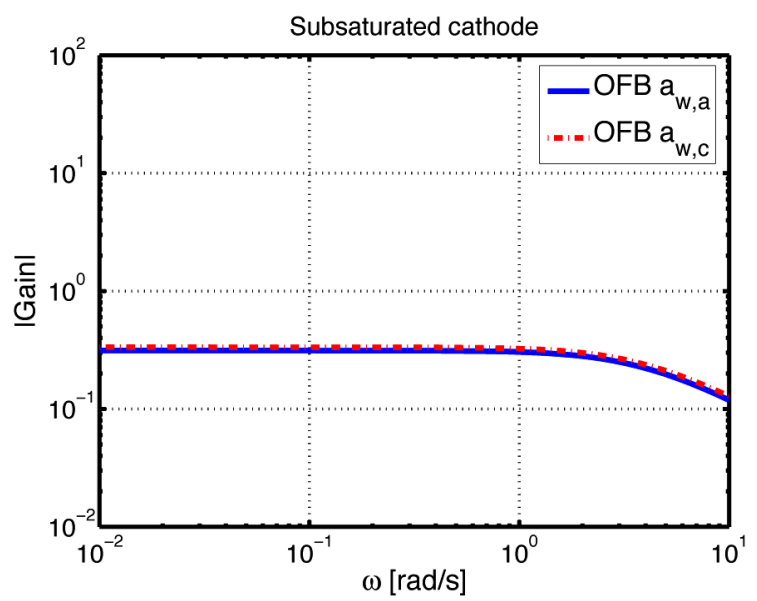

(a)

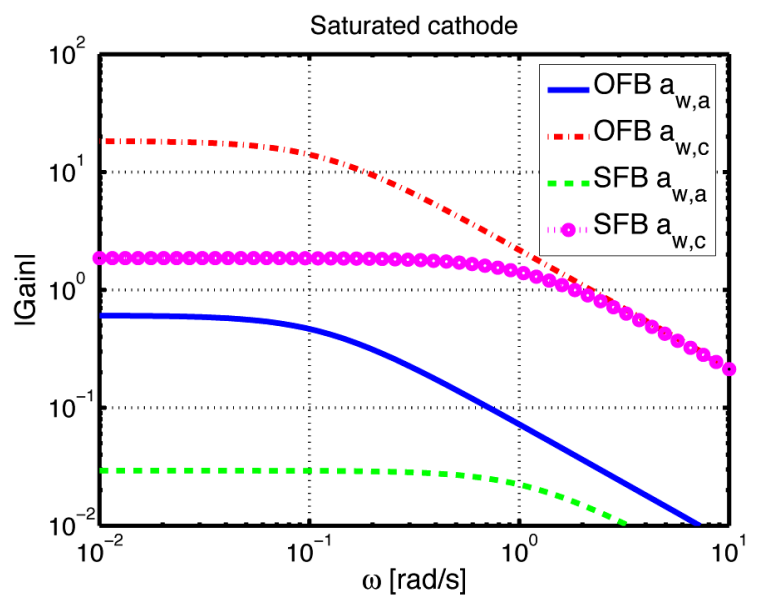

(b)

Fig. 15. Closed-loop sensitivity of anode and cathode water activities to disturbance in the cathode inlet humidity using state feedback and output feedback controllers. Controller gains tuned for subsaturated anode and cathode are used for both (a) subsaturated and (b) saturated conditions.

subsaturated conditions). Therefore, an acceptable regulation of $a_{w, c}$ is obtained by the output feedback controller through the regulation of $a_{w, a}$.

Once the cathode is saturated, the sensitivity of $a_{w, c}$ increases by more than a factor of 30 for the output feedback controller as seen in Fig. 15(b). Even though the disturbance manifests as a large error in $a_{w, c}$, the error in $a_{w, a}$ is comparatively small, as seen from the low sensitivity of $a_{w, a}$. Therefore, the output feedback controller tuned for a condition with $a_{w, c}<1$ does not provide acceptable regulation of the cathode water activity, once $a_{w, c}>1$. Note that when using the full-state feedback controller designed for subsaturated conditions, the closed-loop sensitivity of $a_{w, c}$ to $\phi_{\text {in, } c}$ with a saturated cathode is comparatively smaller, as expected from the transient response shown in Fig. 11.

For the saturated cathode, the difference in the interactions between $a_{w, c}$ and the measured variables is due to the difference in the membrane water transport characteristics. Specifically, the back diffusion component [see (5)] of the membrane water transport depends upon the cathode water content, $\lambda_{c}$. As seen in Fig. 3, the slope of the $\lambda_{c}$ with respect to $a_{w, c}$ becomes more gradual once $a_{w, c}>1$, that is, the increase in the back diffusion is caused by an increase in the mass of water is significantly higher when the water is in vapor phase than when in liquid phase. Consequently the liquid water accumulating in the cathode does not substantially increase the back diffusion and therefore has less influence on the anode water activity. The weaker coupling between the anode and cathode water mass is the main reason for the difference in the open loop dynamics.

\section{Controller Redesign for Saturated Conditions}

The changes in the system dynamic characteristics require a redesign of the output feedback controller for saturated conditions. Using a linear plant obtained by linearizing the plant dynamics with a saturated cathode, we calculate the state feedback gains that minimize the quadratic cost function $J$, defined in (22). As the plant dynamics are different, the parameters $Q$ and $R$ are retuned such that the response of the corresponding optimal output feedback controller satisfies the time-domain performance requirements. This new cost function is then employed to calculate the corresponding optimal output feedback gains using the procedure described in Section V-A.

In this study, the feedback gains for the saturated plant are obtained for the performance metric $J$ with

$$
\begin{aligned}
& Q=\left[\begin{array}{ccc}
20 & 0 & 0 \\
0 & 0.01 & 0 \\
0 & 0 & 0.01
\end{array}\right] \\
& R=\left[\begin{array}{cc}
32 & 0 \\
0 & 100
\end{array}\right] .
\end{aligned}
$$

These values of $Q$ and $R$ are used to calculate the output feedback gains at different operating points, and the resulting gains are shown in Fig. 13 together with the gains designed in Section V-B for subsaturated conditions.

As seen in the fourth column of $K_{y}$ in Fig. 13, the magnitude of the output feedback gains between the error in the anode humidity and the actuators increase significantly when redesigned for a saturated cathode condition. Moreover, these gains increase with a decrease in the slope of $\lambda_{j}$ versus $a_{w, j}$ for $a_{w, j}>1$. Higher controller gains are required under saturated cathode conditions to overcome the poor observability of the cathode water activity from the anode side measurements.

The closed-loop response with a gain-scheduled controller is shown in Fig. 12 by the solid line. For the closed-loop evaluations, the anode humidity sensor with $1 \mathrm{~s}$ time constant is used [14]. The gain scheduling provides a significant improvement in the settling time for $a_{w, c}$ for the step change in the load from 170 to $190 \mathrm{~A}$, and, as seen in Fig. 12, the specified settling time (4s) for water activities is satisfied.

\section{E. Sensitivity To Measurement Noise}

One caveat to applying the high-gain feedback is the increased sensitivity to measurement noise. It should be pointed out that the high gain feedback required for a saturated cathode condition is due to the choice of the measured variables and not the type of controller. Instead of a static feedback controller, a dynamic observer-based state feedback can be tuned such that it achieves the disturbance rejection properties of the full-state feedback controller [see Fig. 14(b)]. The closed-loop sensitivities when using the observers tuned for the subsaturated and saturated cathode conditions on the corresponding 


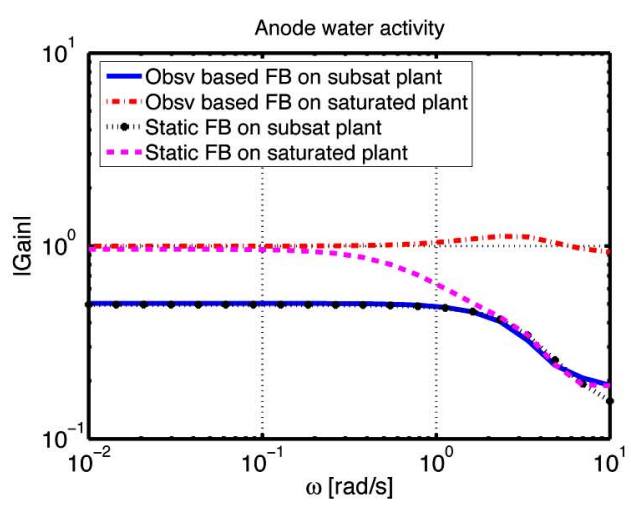

(a)

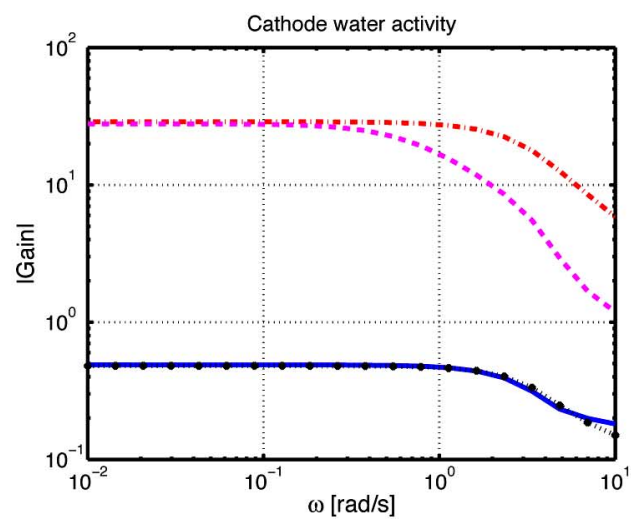

(b)

Fig. 16. Closed loop sensitivity of (a) anode water activity and (b) cathode water activity to measurement noise in $\phi_{a}$.

plants are shown in Fig. 16 by the solid and dashed-dot lines, respectively. A significantly higher sensitivity to noise in anode humidity measurement is seen when using the controller tuned for a saturated condition. High observer gains are obtained corresponding to the cathode water activity which highlights the weak interaction between the cathode water activity and the measured variables under saturated cathode conditions. This weak interaction leads to the inherent tradeoff between disturbance rejection and sensor noise for saturated cathode conditions when using $y=\left[\begin{array}{llll}p_{a} & p_{r} & p_{\mathrm{sm}, a} & \phi_{a}\end{array}\right]^{T}$ for feedback. The sensitivities to sensor noise when using the static output feedback controllers developed in this paper for the sub-saturated and saturated conditions are also shown in Fig. 16 using the dashed-dotted and the dashed lines, respectively.

As the performance requirements for the subsaturated plant $P_{1}$ can be satisfied using the controller designed on the subsaturated model $C_{1}$, the high gain controller designed on the saturated model $C_{2}$ is not required under subsaturated cathode conditions. Fig. 17 compares the closed-loop sensitivity of $a_{w, a}$ and $a_{w, c}$ to noise from anode humidity measurement for four different combinations. These combinations are obtained from implementing the controllers designed for subsaturated and saturated plant models, $C_{1}$ and $C_{2}$, respectively, on a subsaturated plant, $P_{1}$, and on a saturated plant $P_{2}$. One can see that the high-frequency closed-loop sensitivity to sensor noise substantially increases for $C_{2}$, the high gain feedback, thereby requiring a high precision and fast anode humidity measurement. By applying $C_{2}$ only when $a_{w, c}>1$, the gain-scheduled controller

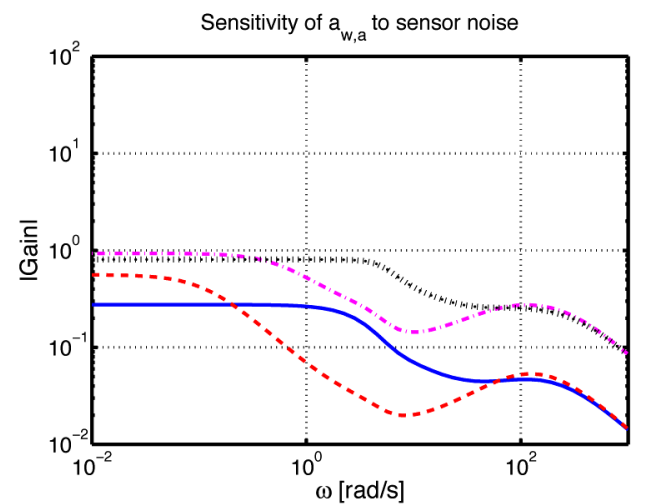

(a)

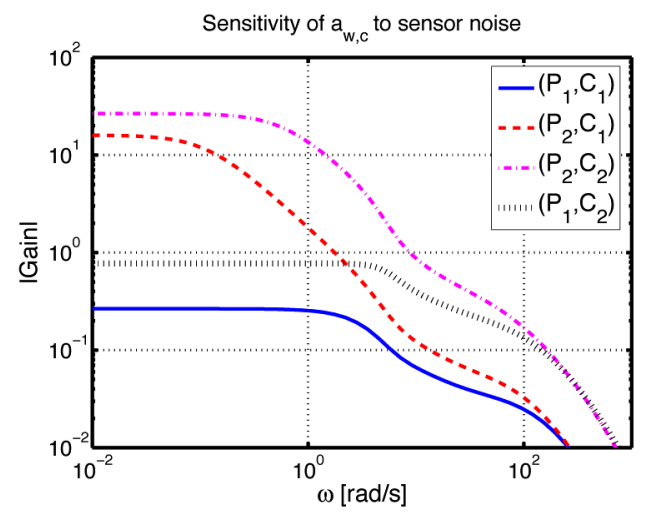

(b)

Fig. 17. Closed-loop sensitivity of (a) anode water activity and (b) cathode water activity to noise in $\phi_{a}$ measurement. The subsaturated plant is denoted by $P_{1}$ and the saturated plant by $P_{2}$. Controllers $C_{1}$ and $C_{2}$ are designed for subsaturated and saturated plant models, respectively.

maintains a lower sensitivity to sensor noise while the cathode is subsaturated and yet satisfies the transient requirements when the cathode is saturated.

The logic used here for switching between controller gains depends upon either the measurement of cathode humidity, or its estimate. Therefore, additional investigation would be required to develop an alternative way to increase the gains independent of cathode humidity measurement.

\section{CONCLUSION}

In this paper, the water management and pressure regulation problem is formulated for a PEMFC system with ejector-based anode recirculation. A desired closed-loop system response to a series of load changes is established using a state feedback controller. A major limitation in implementing this state feedback controller is the need to measure cathode water activity (that is, the level of water in the cathode) in the presence of liquid water in the cathode. When the same controller is evaluated with humidity measurement in place of cathode water activity measurement, the response under saturated cathode conditions slows down substantially.

The transient requirements are shown to be met using a gainscheduled static output feedback controller implemented with three anode side pressure sensors and an anode humidity sensor. Gain scheduling based upon the cathode humidity conditions is required to address the change in: 1) the interaction between 
mass of water in the cathode and the flows in/out of the cathode and 2) the membrane water transport between the anode and cathode. The feedback gains required from the anode humidity sensor to the actuators are significantly higher when the cathode is saturated. As a precaution, we also point out that fast and accurate humidity sensors are essential to achieve the desired performance with the high gain controller.

In this study, the controllers were developed assuming a perfect knowledge of the operating conditions for the fuel cell system. Plant uncertainties, such as changes in the membrane properties over time or variation in the stack temperature, can affect the performance of the resulting control system, and, hence, robust control design techniques should be investigated to guarantee system performance.

\section{ACKNOWLEDGMENT}

The authors would like to thank Prof. J. Freudenberg, Prof. J. Grizzle, and Adjunct Prof. J. Cook of the University of Michigan and J. Koncsol for many constructive discussions.

\section{REFERENCES}

[1] J. Larminie and A. Dicks, Fuel Cell Systems Explained. New York: Wiley, 2003.

[2] J. T. Pukrushpan, A. G. Stefanopoulou, and H. Peng, Control of Fuel Cell Power Systems: Principles, Modeling, Analysis and Feedback Design, ser. Advances in Industrial Control. Berlin, Germany: SpringerVerlag, 2004.

[3] F. N. Büchi and S. Srinivasan, "Operating proton exchange membrane fuel cells without external humidification of the reactant gases," J. Electrochem. Soc., vol. 144, no. 8, pp. 2767-2772, Aug. 1997.

[4] M. Eikerling, A. A. Kornyshev, and A. Kucernak, "Water in polymer electrolyte fuel cell: Friend or foe?," Physics Today, pp. 38-44, Oct. 2006.

[5] P. Rodatz, A. Tsukada, M. Mladek, and L. Guzzella, "Efficiency improvements by pulsed hydrogen supply in PEM fuel cell systems," presented at the 15th IFAC Triennial World Congress, Barcelona, Spain, 2002.

[6] W. Levine and M. Athans, "On the determination of the optimal constant output feedback gains for linear multivariable systems," IEEE Trans. Autom. Control, vol. AC-15, no. 1, pp. 44-8, Feb. 1970.

[7] D. D. Moerder and A. J. Calise, "Convergence of a numerical algorithm for calculating optimal output feedback gains," IEEE Trans. Autom. Control, vol. AC-30, no. 9, pp. 900-903, Sep. 1985.

[8] A. Y. Karnik, J. Sun, and J. H. Buckland, "Control analysis of ejector based fuel cell anode recirculation system," in Proc. Amer. Control Conf., Jun. 2006, pp. 2997-3002.

[9] T. Springer, T. Zawodzinski, and S. Gottesfeld, "Polymer electrolyte fuel cell model," J. Electrochem. Soc., vol. 138, no. 8, pp. 2334-2342, Aug. 1991.

[10] A. Y. Karnik, A. G. Stefanopoulou, and J. Sun, "Water dynamics and requirements using a dynamic and low-order model of a polymer electrolyte fuel cell stack," J. Power Sources, vol. 164, no. 2, pp. 590-605, Feb. 2007.

[11] D. Chen and H. Peng, "Analysis of non-minimum phase behavior of PEM fuel cell membrane humidification systems," in Proc. ACC, Jun. 2005, pp. 3853-3858.

[12] A. Y. Karnik and J. Sun, "Modeling and control of an ejector based anode recirculation system for fuel cells," in Proc. 3rd Int. Conf. Fuel Cell Sci., Eng. Technol., May 2005, pp. 721-731, no. FUELCELL2005-74102.

[13] G. F. Franklin, J. D. Powell, and A. E. Naeini, Feedback Control of Dynamic Systems, 5th ed. Upper Saddle River, NJ: Prentice-Hall, 2005.

[14] [Online]. Available: http://sensirion.com/Sensirion SHT75

[15] J. T. Hinatsu, M. Mizuhata, and H. Takenaka, "Water uptake of perfluorosulfonic acid membranes from liquid water and water vapor," $J$. Electrochem. Soc., vol. 141, no. 6, pp. 1493-1498, Jun. 1994.

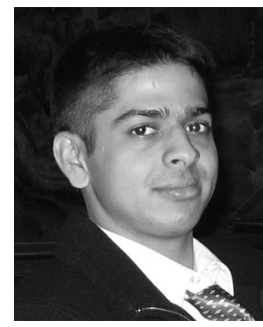

Amey Karnik received the B.Eng. degree from the University of Mumbai, Mumbai, India, in 2002 and the M.S. and Ph.D. degrees from the University of Michigan, Ann Arbor, in 2004 and 2007, respectively. For his doctoral research, he investigated the water management aspect of fuel cell systems.

His research interests include analysis of advanced powertrain systems and application of modern control techniques to automotive problems. $\mathrm{He}$ is currently a Research Engineer with the Company, Dearborn, MI Powertrain Controls R\&A Department, Ford Motor

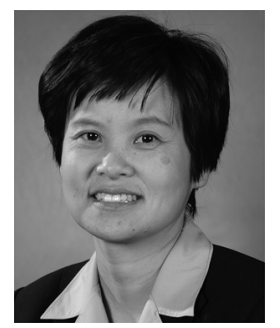

Jing Sun (F'04) received the B.S. and M.S. degrees from the University of Science and Technology of China, Hefei, in 1982 and 1984, respectively, and the $\mathrm{Ph} . \mathrm{D}$. degree from the University of Southern California, Los Angeles, in 1989.

From 1989 to 1993, she was an Assistant Professor with the Electrical and Computer Engineering Department, Wayne State University. She joined Ford Research Laboratory in 1993 where she worked in the Powertrain Control Systems Department. After spending almost ten years in industry, she came back to academia and joined the faculty of the College of Engineering, University of Michigan, Ann Arbor, in 2003 as an Associate Professor. Her research interests include system and control theory and its applications to marine and automotive propulsion systems. She holds over 30 U.S. patents and has coauthored a textbook, Robust Adaptive Control (Prentice-Hall, 1995).

Prof. Sun was one of the three recipients of the 2003 IEEE Control System Technology Award.

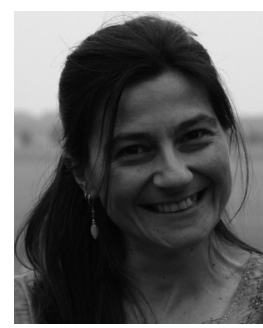

Anna G. Stefanopoulou received the Diploma in naval architecture and marine engineering from the National Technical University of Athens, Athens, Greece in 1991 and the Ph.D. degree in electrical engineering and computer science from the University of Michigan, Ann Arbor, in 1996.

She is presently a Professor with the Mechanical Engineering Department, University of Michigan. She was an Assistant Professor (1998-2000) with the University of California, Santa Barbara, and a Technical Specialist (1996-1997) with the Scientific Research Laboratories, Ford Motor Company. She has coauthored 125 journal and conference articles, which won four Best Paper Awards. She authored the book Control of Fuel Cell Power Systems (Springer, 2004) and holds nine U.S. patents. Her research interests are in multivariable control of internal combustion engines and fuel cell power systems.

Dr. Stefanopoulou is an ASME Fellow, a member of the IEEE Control Systems Society (CSS) Board of Governors, and an Associate Editor of the IEEE TRANSACTIONS ON CONTROL SYSTEM TECHNOLOGY. She was a recipient of the 2005 Outstanding Young Investigator by the ASME DSC division, the 2005 Henry Russel Award, a 2002 Ralph Teetor SAE Educational Award, and a 1997 National Science foundation CAREER Award and was selected as one of the 2002 world's most promising innovators from the MIT Technology Review.

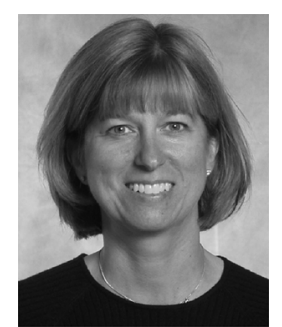

powertrain systems
Julia Buckland received the B.S. and M.S. degrees in aerospace engineering from the University of Cincinnati, Cincinnati, OH, in 1988 and 1993, respectively.

For several years, she worked in the defense industry developing flight control systems for advanced military aircraft. In 1994, she joined Ford Motor Company, Dearborn, MI, where she is currently a Technical Expert in the Powertrain Controls R\&A Department. Her work focuses on modeling and control of advanced technology 